\section{Prostatakarzinom: viele Ärzte, freie Entscheidung?}

Die Befürworter multidisziplinärer Tumorboards bauen auf einen offenen Dialog zwischen Medizinern und Patienten. Das ermögliche eine weniger einseitige Information und eine unabhängige Entscheidung. Aber ist da in der Praxis wirklich etwas dran?

$\mathrm{M}$ oderne Krebskliniken setzen auf multidisziplinäre Zusammenarbeit in der Behandlung ihrer Krebspatienten. Dies soll unter anderem zu einer interaktiven Diskussion führen, wodurch die Voreingenommenheit des einzelnen Arztes weniger ins Gewicht fällt. Aber wirkt es sich tatsächlich positiv aus, wenn etwa Prostatakrebspatienten ihre behandelnden Urologen, Radiologen und Onkologen zugleich treffen, statt sie - wie im niedergelassenen Setting üblich - nacheinander zu konsultieren?

Dieser Frage hat sich eine Studie an 701 Patienten mit niedrigmalignem Prostatakarzinom gewidmet, die im Jahr 2009 an drei Therapiezentren in Boston/USA behandelt wurden. Die Patienten wurden entweder von einem Tumorboard an einer multidisziplinären Postatakarzinomklinik beraten oder von individuellen Ärzten aufeinanderfolgend behandelt. Das primäre Outcome war die Entscheidung der Patienten für eine Active Surveillance.

Die Patienten in den multidisziplinären Kliniken entschieden sich erheblich häufiger (43\%) für Active Surveillance als die in Praxen behandelten Patienten (22\%). Zugleich sank der Anteil der Männer, die eine Prostatektomie oder eine Bestrahlung erhielten, um etwa $30 \%$ (p $<0,001$ ). Signifikant assoziiert mit der Wahl einer Active Surveillance waren ein höheres Alter $(\mathrm{OR}=1,09)$, nicht verheiratet zu sein $(\mathrm{OR}=1,66)$, ein höherer Charlson-Komorbiditätsindex $(\mathrm{OR}=$ $1,37)$ sowie weniger positive Cores (OR $=0,92$ ). Der stärkste Faktor aber war die Konsultation einer multidisziplinären Klinik $(\mathrm{OR}=2,15)$.

Fazit: An multidisziplinär arbeitenden Zentren entscheiden sich Patienten mit nieddrigmalignem Prostatakarzinom signifikant häufiger für Active Surveillance statt für Prostatektomie oder Bestrahlung. Frühere Studien hatten bereits gezeigt, dass Ärzte vor allem jene Behandlungsmodalität empfehlen, die sie selbst anbieten. Diese Art der Beeinflussung könnte eine Behandlung in einem multidisziplinären Zentrum offenbar tatsächlich verhindern.

Christina Berndt

Aizer AA et al. Multidisciplinary care and pursuit of active surveillance in low-risk prostate cancer. J Clin Oncol 2012; 30: 3071-6

\title{
Was bringen Stoßwellen bei erektiler Dysfunktion?
}

Seit mehr als zwei Dekaden ist bekannt, dass die extrakorporale Stoßwellentherapie die Angiogenese fördert. Für israelische Urologen lag es nahe, das Verfahren auch bei erektiler Dysfunktion zu prüfen. Sie konnten zeigen, dass aufgrund dieser gut verträglichen Behandlung signifikant mehr Männer wieder mit ihrem Geschlechtsleben zufrieden sind als Männer, bei denen die Stoßwellentherapie nur vorgetäuscht wurde.

sraelische Urologen hatten in einer Pilotstudie die extrakorporale Stoßwellentherapie (ESWT) erfolgreich bei Patienten mit erektiler Dysfunktion eingesetzt. In eine randomisierte Doppelblindstudie wurden 67 heterosexuelle Männer mit ED aufgenommen, die zuvor auf eine Behandlung mit einem oralen Phosphodiesterase (PDE)-5-Hemmer angesprochen hatten. Das Präparat wurde einen Monat vor Studienbeginn abgesetzt, dann erhielten die Männer entweder eine ESWT oder eine Scheinbehandlung, bei der zwar auch ein Schallkopf verwendet, die ESWT selbst aber nur vorgetäuscht wurde. Die ESWT erfolgte jeweils an fünf Stellen des Penis durch 300 Stoßwellen mit einer Energiedichte von nur 0,09 mJ/ $\mathrm{mm}^{2}$ über einen Zeitraum von $15 \mathrm{Minu}-$ ten. Behandelt wurde insgesamt neun Wochen lang mit zwei Anwendungen pro Woche und drei Wochen Pausen nach den ersten drei Therapiewochen. Wegen der geringen Energie sei eine systemische oder lokale Analgesie nicht erforderlich gewesen, so die Autoren.

Zur Beurteilung der Erektionsstörung verwendeten die Urologen den IIEF (,international index of erectile function")Score mit maximal 30 Punkten. Mit der ESWT wurden zwei Wochen nach der Behandlung signifikant mehr Punkte erreicht als in der Vergleichsgruppe: 19 Patienten, bei denen vor der Therapie kein Geschlechtsverkehr klappte, waren nach der Behandlung in ihrem Bemühen wieder erfolgreich. In der Kontrollgruppe war das bei keinem Patienten der Fall. Der maximale postischämische Blutfluss im Penis lag in der ESWT-Gruppe mit 8,2 vs. $0,1 \mathrm{ml} / \mathrm{min} / \mathrm{dl}$ ebenfalls signifikant höher als bei Männern der Vergleichsgruppe.

Fazit: Nach einer ESWT waren signifikant mehr Männer wieder mit ihrem Liebesleben zufrieden als Männer, die nur eine Scheinbehandlung erhielten. Allerdings räumen die Autoren ein, dass die Verbesserung im IIEF-Score nach der ESWT nicht so stark war wie die nach Einnahme eines PDE-5-Hemmers in den ersten Studien zur pharmakologischen ED-Therapie Ende der 1990er-Jahre. Peter Leiner

Vardi Y et al. Does Low Intensity Extracorporeal Shock Wave Therapy Have a Physiological Effect on Erectile Function? Short-Term Results of a Randomized, Double-Blind, Sham Controlled Study. J Urol 2012; 187: 1769-75 